\title{
Niveles de IgA en adultos con enfermedad celíaca
}

\author{
Silvia Ferreira ${ }^{1}$, María Elena Chamorro², José Masi' ${ }^{2}$, Diana Sanabria ${ }^{1}$, Sara Benegas ${ }^{1}$, María Mercedes

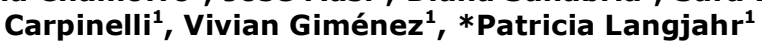 \\ ${ }^{1}$ Universidad Nacional de Asunción, Instituto de Investigaciones en Ciencias de la Salud, Departamento de \\ Inmunología, San Lorenzo, Paraguay \\ ${ }^{2}$ Fundación Paraguaya de Celíacos, Asunción, Paraguay
}

Cómo referenciar este artículo/
How to reference this article:
Ferreira S, Chamorro ME, Masi J, Sanabria D, Benegas S, Carpinelli MM, et al. Niveles de IgA en adultos con enfermedad celíaca. Mem. Inst. Investig. Cienc. Salud. 2019; 17(1):54-58

\begin{abstract}
RES U MEN
La enfermedad celíaca (EC) es una enfermedad autoinmune sistémica desencadenada por el consumo de gluten de la dieta en personas con susceptibilidad genética. Los principales test serológicos utilizados para el diagnóstico y seguimiento de la EC son pruebas basadas en anticuerpos de isotipo inmunoglobulina ( $\mathrm{Ig}$ ) A, siendo la determinación de IgA antitransglutaminasa tisular (tTG)2 la prueba serológica inicial de elección. La deficiencia selectiva de IgA (DSIgA), es más prevalente en pacientes con EC que en la población general, dificultando el diagnostico serológico de la enfermedad. En el presente estudio observacional descriptivo, se incluyeron 74 pacientes adultos con diagnóstico confirmado de EC y se determinó IgA anti-tTG2 en suero mediante ensayo de ELISA a fin de detectar a aquellos pacientes con niveles indeterminados o negativos, los cuales podrían presentar DSIgA. Se dosó IgA total en el suero de estos pacientes por inmunodifusión radial y el promedio fue de $237,8 \pm 100,6 \mathrm{mg} / \mathrm{dL}$. En una paciente del sexo femenino fue detectada IgA total menor a $7 \mathrm{mg} / \mathrm{dL}$, con niveles séricos de IgG e IgM normales, característicos de la DSIgA. Así, la frecuencia calculada de DSIgA fue de $1,35 \%$ en la población con EC estudiada. En conclusión, este trabajo es una primera aproximación para describir la frecuencia de DSIgA en pacientes con EC del país y reafirma la importancia de incluir el dosaje de IgA total en el caso de realizar test serológicos de la EC basados en IgA.
\end{abstract}

Palabras clave: Enfermedad celíaca, deficiencia de IgA, autoinmunidad.

\section{IgA levels in adults with celiac disease}

\section{ABSTRACT}

Celiac disease (CD) is a systemic autoimmune disease, which is triggered by gluten intake in genetically susceptible individuals. The most important serological markers used for CD diagnosis are test based on immunoglobulin (Ig) A isotype, being the determination of tissue anti-transglutaminase IgA (tTG) 2 the marker of choice for diagnosis. Selective IgA deficiency (SIgAD) is more prevalent in CD patients than in the general population, making the serologically diagnosis of the diseasedifficult. In the present descriptive observational study, 74 adult patients with a confirmed CD diagnosis were included and anti-tTG2 IgA was determined by using ELISA assay in order to detect those patients with undetermined or negative levels, which could present SIgAD. The total IgA level was determined in these patients and the mean concentration was $237.8 \pm 100.6 \mathrm{mg} / \mathrm{dL}$. In a female patient, total IgA was less than $7 \mathrm{mg} / \mathrm{dL}$, with normal IgG and IgM levels, characteristic of SIgAD. Thus, the calculated frequency of SIgAD was $1.35 \%$ of the CD study population. In conclusion, this work is a first approach to describe the frequency of SIgAD in CD patients of the country and reaffirms the importance of including total IgA determination in the case of conducting IgA-based serological tests specific for CD.

Keywords: Celiac disease, IgA deficiency, autoimmunity. 


\section{INTRODUCCIÓN}

La enfermedad celíaca (EC) es una enfermedad autoinmune desencadenada por el consumo de gluten de la dieta en personas con susceptibilidad genética, que afecta principalmente el intestino delgado ${ }^{(1,2)}$. La prevalencia de la EC en población norteamericana es de aproximadamente $1 \%{ }^{(3)}$. El principal factor genético involucrado es el haplotipo de antígeno leucocitario humano (HLA) DQ2/DQ8 ${ }^{(1,4)}$.

El diagnóstico de la EC en pacientes adultos se realiza utilizando una combinación de pruebas serológicas y biopsia intestinal en pacientes que presentan una dieta conteniendo gluten ${ }^{(5)}$. Los anticuerpos IgA anti-transglutaminasa tisular (tTG)2, IgA anti-endomisio (IgAEMA) e IgA anti-péptidos de gliadina desaminados (DGP) son marcadores serológicos de diagnóstico ampliamente utilizados. La prueba inicial de elección para el diagnóstico en pacientes con sospecha clínica es la determinación de IgA anti-tTG2, ya que este autoanticuerpo es altamente sensible y específico (mayor al 95\%) para el diagnóstico de la $E C^{(2,6)}$. Además, en el seguimiento de pacientes con $E C$ en tratamiento con dieta libre de gluten (DLG) se utilizan test serológicos basados en IgA (IgA anti-tTG2 y anti-DGP) a fin de evaluar el cumplimiento dietario(7).

La deficiencia selectiva de IgA (DSIgA) es la inmunodeficiencia primaria más frecuente en la población general, con incidencias que va de 1:163 a 1:965 (menor al 1\%), dependiendo de la población ${ }^{(8,9)}$. De acuerdo a la definición actual, se considera DSIgA cuando se presenta IgA en suero menor a $7 \mathrm{mg} / \mathrm{dL}(0,07 \mathrm{~g} / \mathrm{L})$, con concentraciones séricas de los isotipos de inmunoglobulinas IgM e IgG normales, en individuos de 4 años o mayores ${ }^{(8,10)}$. La mayoría (dos tercios) de los individuos con DSIgA son asintomáticos, sin embargo, puede presentarse asociada a infecciones recurrentes del tracto respiratorio y gastrointestinal y a ciertas enfermedades autoinmunes ${ }^{(8,11)}$.

La DSIgA es más prevalente en pacientes con EC que en la población general

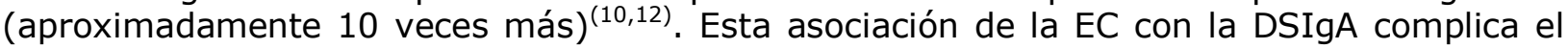
diagnostico serológico de la enfermedad. Así, los pacientes con DSIgA no producen IgA antitTG2 ni IgA-EMA, lo cual puede llevar a resultados falsos negativos durante la realización de test serológicos para el diagnóstico de la patología(13). Por lo tanto, en pacientes con sospecha de EC se sugiere la cuantificación de IgA total, en conjunto con los anticuerpos IgA anti-tTG2 y IgA-EMA. En casos de DSIgA se recomienda la determinación de anticuerpos IgG anti-tTG2 y/o anti-DGP( ${ }^{(6)}$.

A su vez, se ha reportado que pacientes con DSIgA presentan $6,5 \%$ de prevalencia de la $E C^{(14)}$, demostrando una asociación entre ambas entidades.

En el país no se cuentan con datos sobre la prevalencia de DSIgA en pacientes con EC. EI presente estudio tuvo como objetivo determinar el nivel de IgA total en pacientes adultos con EC que se sometieron a dosaje de IgA anti-tTG2 y evaluar la frecuencia de DSIgA en estos pacientes de la población paraguaya estudiada.

\section{PACIENTES Y MÉTODOS \\ Pacientes}

El presente estudio es observacional descriptivo, de corte transverso. Se incluyeron a 74 pacientes adultos (con 18 años cumplidos o mayor), de ambos sexos, diagnosticados con EC por un médico especialista. Los pacientes fueron reclutados con la colaboración de la Fundación Paraguaya de Celíacos (FUPACEL) durante el periodo 2015-2017 y se testaron para IgA anti-transglutaminasa. De ellos, 23 eran pacientes con diagnóstico reciente, sin DLG (no tratados o EC activa) y 51 eran pacientes con DLG (con un mínimo de 6 meses de duración de la dieta). A todos los participantes se les realizó un cuestionario a fin de recoger información sobre sus características demográficas y clínicas. Se excluyeron pacientes con cáncer o embarazo (criterios de exclusión). Se obtuvieron muestras de suero de los pacientes y se conservaron a $-20^{\circ} \mathrm{C}$ hasta el momento del análisis.

\section{Consideraciones éticas}

El presente estudio fue aprobado por el Comité Científico y de Ética del Instituto de Investigaciones en Ciencias de la Salud, Universidad Nacional de Asunción, San Lorenzo, Paraguay. Se procedió a la toma de muestra en aquellos pacientes que accedieron a participar de manera voluntaria y firmaron un consentimiento informado escrito.

\section{Determinación de niveles de IgA anti-tTG2}

Los niveles séricos de anticuerpos IgA anti-tTG2 se determinaron utilizando un ensayo de inmunoabsorción ligado a enzima (ELISA) (Aeskulisa, Wendelsheim, Alemania), según las 
instrucciones del fabricante. Las muestras fueron diluidas empleando el factor de dilución 1:101 recomendado por el fabricante. Los niveles séricos de anticuerpos IgA anti-tTG2 se clasificaron en positivos ( $>18 \mathrm{U} / \mathrm{mL}$ ), indeterminados $(12-18 \mathrm{U} / \mathrm{mL}$ ) y negativos $(<12$ $\mathrm{U} / \mathrm{mL}$ ), de acuerdo con las especificaciones del kit.

\section{Determinación de Ig}

En aquellos pacientes que presentaron IgA anti-tTG2 indeterminado o negativo se determinó IgA total en suero utilizando inmunodifusión radial cuantitativa (IDR) (DiffuPlate, Biocentífica, Buenos Aires, Argentina). En caso de niveles no detectados de IgA con el método citado, se confirmó utilizando IDR de baja concentración (límite de detección 1,8 $\mathrm{mg} / \mathrm{dL}$ ), en duplicado. IgM e IgG en suero se determinaron utilizando el método de IDR.

\section{Análisis estadístico}

Los datos fueron almacenados en planillas del programa Excel v. 2007. Se estimaron medias y desviaciones estándar; se calcularon la frecuencia simple y porcentajes de las variables analizadas.

\section{RESULTADOS}

Un total de 74 pacientes adultos con diagnóstico de EC (57 mujeres y 17 hombres) fueron incluidos en este estudio, a los que se les realizó dosaje de IgA anti-tTG2, a fin de detectar aquellos que presentaban IgA anti-tTG2 indeterminado o negativo, los cuales podrían presentar DSIgA. De ellos, 23 presentaban EC activa (sin DLG) y 51 pacientes se encontraban con tratamiento con DLG, con una duración promedio de la DLG de 5,6 años (Tabla 1). Los pacientes sin DLG presentaron anticuerpo IgA anti-tTG2 positivo (100\%); de los pacientes con DLG presentaron IgA anti-tTG2 positivo $27,5 \%$, negativo $64,7 \%$ e indeterminado $7,8 \%$.

Tabla 1. Características demográficas de los sujetos de estudio.

\begin{tabular}{lcc}
\hline & EC sin DLG & EC con DLG \\
\hline Número de participantes & 23 & 51 \\
Edad promedio, años \pm DE & $35,6 \pm 12,0$ & $36,0 \pm 12,9$ \\
Sexo, $\mathbf{n}(\%)$ & & \\
F & $16(69,6)$ & $41(80,4)$ \\
$\mathbf{M}$ & $7(30,4)$ & $10(19,6)$ \\
\hline
\end{tabular}

EC, enfermedad celíaca. DLG, dieta libre de gluten. DE, desviación estándar. Edad mínima/máxima: EC sin DLG 19/65 años y EC con DLG 19/67 años.

En aquellos pacientes que presentaban IgA anti-tTG2 indeterminado o negativo se determinó el nivel sérico de IgA total, a fin de excluir deficiencia de IgA total. El promedio de IgA observado fue de $237,8 \pm 100,6 \mathrm{mg} / \mathrm{dL}$ (Tabla 2). IgA menor a $7 \mathrm{mg} / \mathrm{dL}$, característico de la DSIgA, fue detectado en una paciente del sexo femenino que presentaba IgA anti-tTG2 negativo y DLG hace 4 años. Además, en esta paciente fueron dosadas IgM e IgG total, encontrándose ambas Ig dentro del rango normal (Tabla 3), en coincidencia con una DSIgA. Todos los demás pacientes presentaron niveles de IgA total dentro del rango normal para la edad.

Tabla 2. Nivel de IgA total en pacientes con EC que presentaban IgA anti-tTG2 indeterminado o negativo.

\begin{tabular}{ll}
\hline & Valor \\
\hline IgA total (mg/dL)*, promedio \pm DE & $237,8 \pm 100,6$ \\
Deficiencia de IgA, & $1 * *$ \\
\hline
\end{tabular}

IgA, inmunoglobulina A. tTG2, transglutaminasa tisular 2. DE, desviación estándar. *Rango de referencia: $90-400 \mathrm{mg} / \mathrm{dL}, * * I g A$ total $<1,8 \mathrm{mg} / \mathrm{dL}$. 
Tabla 3. Niveles de inmunoglobulinas en paciente con EC y deficiencia de IgA total.

\begin{tabular}{lc}
\hline & $\begin{array}{c}\text { Niveles de Ig séricas } \\
(\mathbf{m g} / \mathbf{d L})\end{array}$ \\
\hline IgA total* & $<1,8$ \\
IgG total** & 1002,4 \\
IgM total*** & 142 \\
\hline
\end{tabular}

Ig, inmunoglobulina. *Rango de referencia: $90-400 \mathrm{mg} / \mathrm{dL} ; * *$ Rango de referencia: $600-1650 \mathrm{mg} / \mathrm{dL}$; $* * *$ Rango de referencia: $75-300 \mathrm{mg} / \mathrm{dL}$.

Considerando la totalidad de pacientes con EC testeados para IgA anti-tTG2 $(n=74)$ y que se detectó 1 caso de DSIgA, la frecuencia calculada es de 1,35\% de DSIgA en la población con EC estudiada.

\section{DISCUSIÓN}

Este trabajo es una primera aproximación para describir la frecuencia de DSIgA en pacientes con diagnóstico de EC del país. Se incluyeron pacientes adultos con EC que fueron sometidos a dosaje de IgA anti-tTG2, siendo el mayor porcentaje del sexo femenino, en concordancia con una mayor prevalencia de la enfermedad en mujeres que en hombres ${ }^{(2)}$.

En el país no se cuentan con datos sobre la prevalencia de DSIgA en pacientes con EC ni en la población general. En este trabajo, se encontró una frecuencia de 1,35\% de DSIgA en la población de pacientes adultos con EC estudiada. Esta frecuencia es similar a lo reportado en un estudio donde se incluyeron pacientes con diagnostico confirmado de EC de Norteamérica $(1,9 \%)^{(15)}$ y más baja a la reportada en población europea $(2,6 \%)^{(16)}$. Esto puede deberse a factores tales como diferencias genéticas de las poblaciones o al tamaño de la población estudiada. Por otro lado, la frecuencia observada de DSIgA en EC en este estudio es mayor a la reportada en otros estudios donde incluyeron pacientes con sospecha de EC que se sometieron a screening por test serológicos ${ }^{(17)}$, a diferencia de este trabajo donde se incluyeron pacientes con EC con diagnóstico confirmado por un especialista.

La frecuencia de DSIgA en pacientes con EC observada en este trabajo es mayor a la reportada en población sana(15), en coincidencia a lo descrito de que la prevalencia de DSIgA es mayor en EC que en la población en general ${ }^{(18)}$. Se sugiere que factores genéticos están involucrados tanto en el desarrollo de la EC como en la DSIgA ${ }^{(8)}$. Estos datos sugieren la importancia de considerar la mayor frecuencia de DSIgA en pacientes con EC de nuestro país, ya que esta inmunodeficiencia puede complicar el diagnóstico de la EC, así como el seguimiento de los pacientes utilizando test serológicos.

En este trabajo se incluyeron pacientes adultos con EC. Se ha reportado que el aumento de prevalencia de la DSIgA en la EC no está influenciado por la edad(16), por lo que la frecuencia de DSIgA descrita podría ser similar en pacientes pediátricos con EC.

Una limitante del trabajo, es el número relativamente pequeño de pacientes con EC incluidos. Sin embargo, representa un número considerable teniendo en cuenta la prevalencia estimada del $1 \%$ de la patología, el número relativamente bajo de habitantes de nuestro país y que todos los pacientes incluidos presentaban diagnóstico confirmado de EC por un especialista, a diferencia de otros trabajos donde se incluyen casos de sospecha de $E C^{(17)}$. Futuros estudios de prevalencia de DSIgA en la EC son relevantes a nivel del país, donde se pueden incluir un mayor número de pacientes con EC; además es importante abordar en el futuro el estudio de la prevalencia de DSIgA en población general del país.

En conclusión, la frecuencia observada en este estudio es similar a la reportada en otras poblaciones, reafirmando la importancia de incluir el dosaje de IgA total en el caso de realizar test serológicos basados en IgA tanto para el diagnóstico como seguimiento de la EC.

Conflicto de intereses. Los autores declaran no tener ningún conflicto de intereses.

\section{REFERENCIAS BIBLIOGRAFICAS}

1. Lebwohl B, Sanders DS, Green PHR. Coeliac disease. Lancet. 2018;391(10115):70-81.
2. Fasano A, Catassi C. Celiac disease. N Engl J Med. 2012; 367(25):2419-26. 
3. Choung RS, Larson SA, Khaleghi $S$, Pharm $D$, Ovsyannikova IG, King $\mathrm{KS}$, et al. Prevalence and Morbidity of Undiagnosed Celiac Disease From a Community-based Study. 2018;152(4):830-9.

4. Sollid L. Triggers and drivers of autoimmunity: lessons from coeliac disease. Nat Rev Immunol. 2013;13(4):1-18.

5. Rubio-Tapia A, Hill I, Kelly CP, Calderwood $\mathrm{AH} M \mathrm{MJ}$ American College of Gastroenterology Clinical Guideline: Diagnosis and Management of Celiac Disease. Am J Gastroenterol. 2013;108 (5):656-77.

6. Husby S, Murray JA. Diagnosing coeliac disease and the potential for serological markers. Nat Rev Gastroenterol Hepatol. 2014;11(11):655-63.

7. Sharkey LM, Corbett G, Currie E, Lee J, Sweeney N, Woodward JM. Alimentary Pharmacology and Therapeutics Optimising delivery of care in coeliac disease comparison of the bene $\mathrm{fi}$ ts of repeat biopsy and serological follow-up. Aliment Pharmacol Ther. 2013;38(10):1278-91.

8. Yazdani R, Azizi G, Abolhassani $H$, Aghamohammadi A. Selective IgA Deficiency: Epidemiology, Pathogenesis, Clinical Phenotype, Diagnosis, Prognosis and Management. Scand J Immunol. 2017;85(1):3-12.

9. Picard C, Gaspar HB, Al-herz W, Bousfiha A, Sullivan KE. International Union of Immunological Societies: 2017 Primary Immunodeficiency Diseases Committee Report on Inborn Errors of Immunity. J Clin Immunol. 2018;38(1):96-128.

10. Notarangelo LD, Fischer A, Geha RS, Casanova JL, Chapel $H$, Conley ME, Cunningham-Rundles $\mathrm{C}$, Etzioni A $\mathrm{HL}$. International Union of Immunological
Societies

Immunodeficiencies

(IUIS)

Committee ] 2010;124(6):1161-78

11. Latiff AHA, Kerr MA. The clinical significance of immunoglobulin A deficiency. Ann Clin Biochem. 2007;44(2):131-9.

12. Wang $N$, Shen $N$, Vyse TJ, Anand V, Gunnarson I, Sturfelt G, et al. Selective IgA Deficiency in Autoimmune Diseases. Mol Med. 2011;17(11-12):1383-96.

13. Giorgio $F$, Principi $M$, Losurdo $G$, Piscitelli $D$, Iannone A, Barone $M$, et al. Seronegative Celiac Disease and Immunoglobulin Deficiency: Where to Look in the Submerged Iceberg? Nutrients. 2015;7(9): 7486-504.

14. Ludvigsson JF, Neovius M HL. Association Between IgA Deficiency \& Other Autoimmune Conditions: A PopulationBased Matched Cohort Study. J Clin Immunol. 2014;34(4):444-51.

15. Pallav K, Xu H, Leffler DA, Kabbani T, Kelly CP. Immunoglobulin A deficiency in celiac disease in the United States. J Gastroenterol Hepatol. 2016;31(1):133-7.

16. Cataldo F, Marino V, Ventura A, Bottaro G, Corazza GR. Prevalence and clinical features of selective immunoglobulin A deficiency in coeliac disease: an Italian multicentre study. Italian Society of Paediatric Gastroenterology and Hepatology (SIGEP) and "Club del Tenue" Working Groups on Coeliac Disease. Gut. 1998;42(3):362-5.

17. McGowan KE, Lyon ME, Butzner JD. Celiac disease and IgA deficiency: Complications of serological testing approaches encountered in the clinic. Clin Chem. 2008;54(7):1203-9.

18. Yel L. Selective IgA deficiency. J Clin Immunol. 2010;30(1):10-6. 